\title{
Bayesian Detection of Coding Regions in DNA/RNA Sequences Through Event Factoring
}

\author{
Renatha Oliva Capua ${ }^{1}$, Helena Cristina da Gama Leitão ${ }^{1}$, and Jorge Stolfi ${ }^{2}$ \\ ${ }^{1}$ Institute of Computing, Federal Fluminense University (UFF) \\ Rua Passo da Pátria, 156, Bloco E - 24210-240 Niterói, RJ - Brazil \\ \{rcapua, hcgl\}@ic.uff.br \\ ${ }^{2}$ Institute of Computing, University of Campinas (UNICAMP) \\ Cx. Postal 6176 - 13081-970 Campinas, SP - Brazil \\ stolfi@ic.unicamp.br
}

\begin{abstract}
We describe a Bayesian inference method for the identification of protein coding regions (active or residual) in DNA or RNA sequences. Its main feature is the computation of the conditional and $a$ priori probabilities required in Bayes's formula by factoring each event (possible annotation) for a nucleotide string into the concatenation of shorter events, believed to be independent. The factoring allows us to obtain fast but reliable estimates for these parameters from readily available databases; whereas the probability estimation for unfactored events would require databases and tables of astronomical size. Promising results were obtained in tests with natural and artificial genomes.
\end{abstract}

Keywords: coding regions, ab-initio DNA tagging, Bayesian inference.

\section{Introduction}

We describe here a new statistical inference method for finding protein-coding regions in genomes. Our method is well-grounded on Bayesian inference theory, and is easily adapted to different genome models.

\subsection{Coding and Non-coding Regions}

The protein-coding regions of a genome specify the sequence of aminoacids in some protein, according to the well-known protein genetic code [1. A coding region is active if it is still transcribed by the organism in appropriate circumstances. Such regions comprise only a fraction of an organism's genome - varying from almost $100 \%$ in viruses, prokaryotes, and archaea, to $10 \%$ or less in eukaryotes. Another important fraction of the genome, which may be dominant in eukaryotes, consists of fossil coding regions - copies of active coding regions of some ancestral genome, which have become inactive or un-transcribable due to mutations and truncations. These fossil regions may be embedded in any non-functional part of the genome, such as between genes and in the introns of eukaryotes. Finally, genomes are believed to contain junk regions that are 
the result of "accidental" events — such as single-base insertions and duplication of pre-existing junk sequences. The primary goal of a genome classification algorithm is to identify the protein-coding regions of a given genome.

\subsection{Homology-Based Methods}

The most successful genome classifiers are the homology-based methods, that rely on the detection of known gene expression clues (operons, mRNA splicing markers) and comparison with previously identified bio-sequences, from the same organism or from other organisms [2].

By their own nature, homology-based methods can identify only coding regions that are still active, or that can still be recognized (by similarity) as descendants of active regions in some ancestral genome. They cannot distinguish truly junk regions from fossil coding regions which are not similar to any known active region. Homology methods may also fail to identify an active coding region if its nucleotide sequence is "new to science" (not represented in available databases) - a situation which is not as rare as one may think 3].

\subsection{Ab-Initio Methods}

To distinguish fossil coding regions from junk and other types of DNA, one must resort to ab initio genome classifiers - algorithms that rely only on the fact that protein-coding regions, being constrained to represent useful and functioning proteins, have different local statistics than other parts of the genome.

The ab-initio approach imposes certain limitations on the performance and results of the classifier. Being based on statistical (rather than logical) inference, the result too is usually probabilistic rather than categorical, and subject to mistakes due to freak coincidences. Moreover, since the differences between coding and non-coding regions are small, one must analyze relatively long segments of the genome in order to get confident and accurate classifications. For this reason, ab-initio methods cannot be expected to detect very short coding or non-coding regions, or locate the exact transition point between two adjacent regions.

\subsection{Previous Work}

There are many ab-initio classifiers described in the literature [4], but none is definitely better than all the others 5]. They use various mathematical models and algorithms, such as neural networks [6, signal processing [7, and Markov models (generally between $2^{\text {nd }}$ and $5^{\text {th }}$ order) [8]. While all those classifiers rely on statistical differences between coding and non-coding regions, their statistical models and inference rules are often ad-hoc and/or hidden in an inscrutable computation model. Methods that explicitly use statistical inference include those of Fickett [3] and Staden and McLahan [9].

\subsection{Outline of Method}

Our algorithm breaks the input sequence into overlapping windows, typically 10 to 100 bases long, and uses Bayesian inference to determine the probability that 
each window belongs to a coding region or to a non-coding region, or contains a transition between two such regions.

Direct estimation of the conditional probabilities required by Bayes's formula, with any useful accuracy, would require labeled databases ("training sets") and tables of astronomical size. Our algorithm avoids the problem by factoring each possible labeling of the window into a sequence of short events, whose probabilities can be easily and accurately estimated from existing databases [10].

\section{Definition of the Problem}

\subsection{Genome Model}

In our model, the classifier's input is a DNA sequence, represented as a string $b=$ $\left(b_{0}, b_{1}, \ldots, b_{n-1}\right)$ where each $b_{i}$ is a letter from the base alphabet $\mathcal{B}=\{\mathrm{A}, \mathrm{T}, \mathrm{C}, \mathrm{G}\}$. The string $b$ is assumed to be the concatenation of several non-empty regions, each being either coding or non-coding. We assume furthermore that each coding region derives from a gene, a concatenation of three-letter codons. However, in order to account for mRNA editing in active coding regions, and accidental truncations in fossil coding regions, we allow that a coding region of the input string may be an arbitrary substring of its original gene.

\subsection{Genome Labelings}

A (full) labeling of the input string is another string $e=\left(e_{0}, e_{1}, \ldots, e_{n-1}\right)$, with the same length $n$, where each $e_{i}$ is a letter from the label alphabet $\mathcal{E}=\{\mathrm{N}, \mathrm{D}, \mathrm{E}, \mathrm{F}\}$. In the true or correct labeling, the label $e_{i}$ is $\mathrm{N}$ if nucleotide $b_{i}$ belongs to a noncoding region; otherwise it is $\mathrm{D}, \mathrm{E}$, or $\mathrm{F}$ according to whether $b_{i}$ descends from the first, second, or third base, respectively, of a codon in the original gene. Needless to say, the true labeling of a natural genome is usually unknown (and, in fact, essentially unknowable).

A full labeling $e$ of the input string implies a weak labeling $c=\left(c_{0}, c_{1}, \ldots, c_{n-1}\right)$, where each $c_{i}$ is $\mathrm{N}$ if $e_{i}$ is $\mathrm{N}$, and $\mathrm{K}$ (meaning "a coding base") if $e_{i}$ is D, E, or F. A full labeling also implies a frame labeling $\phi=\left(\phi_{0}, \phi_{1}, \ldots, \phi_{n-1}\right)$, where each $\phi_{i}$ is either '-', 'o', '+' or '?', depending on the full label $e_{i}$ and the value of $i^{*}=i \bmod 3$ according to the table at right.

Table 1 .

\begin{tabular}{|c|c|c|c|c|}
\hline & \multicolumn{4}{|c|}{$e_{i}$} \\
\hline$i^{*}$ & $\mathrm{D}$ & $\mathrm{E}$ & $\mathrm{F}$ & $\mathrm{N}$ \\
\hline 0 & $\circ$ & + & - & $?$ \\
1 & - & $\circ$ & + & $?$ \\
2 & + & - & $\circ$ & $?$ \\
\hline
\end{tabular}

Note that the frame label is constant $(-$, o, or +$)$ within each coding region, and specifies one of the three possible codon reading frames [1] for that region.

\subsection{Window Probabilities and Ergodicity}

We define a window of the input genome as any finite set of consecutive indices $w=\{r, r+1 \ldots, s\} \subseteq\{0,1, \ldots, n-1\}$. The size of that window is $|w|=s-r+1$; 
its content is the basis sequence $b[w]=\left(b_{r}, b_{r+1}, \ldots, b_{s}\right)$; and its true labeling is the label sequence $e[w]=\left(e_{r}, e_{r+1}, \ldots, e_{s}\right)$.

We assume that the input genome is ergodic; in the sense that, considering all possible input genomes, the probabilities $\operatorname{Pr}(b[w]=\beta \wedge e[w]=\eta)$, for each window $w$ and each pair $\beta \in \mathcal{B}^{|w|}$ and $\eta \in \mathcal{E}^{|w|}$, depend only on $\eta$ and $\beta$ (and on the window size $|w|)$, but not on the position of the window $w$. Then we can write simply $\operatorname{Pr}(\eta)=\operatorname{Pr}(e[w]=\eta), \operatorname{Pr}(\beta)=\operatorname{Pr}(b[w]=\beta), \operatorname{Pr}(\eta \mid \beta)=\operatorname{Pr}(e[w]=$ $\eta \mid b[w]=\beta)$, and so on, where $w$ is any window of size $|\beta|=|\eta|$.

\subsection{Classifier Output}

The goal of our algorithm is to compute, for each input base $b_{i}$, four real numbers $\mathrm{P}[i, \mathrm{~N}], \mathrm{P}[i, \mathrm{D}], \mathrm{P}[i, \mathrm{E}]$, and $\mathrm{P}[i, \mathrm{~F}]$, where $\mathrm{P}[i, \varepsilon]$ is the probability that $\varepsilon$ is the true full label of input base $b_{i}$. Note that from this data one can compute the probability that base $b_{i}$ belongs to a coding region, namely $\mathrm{P}[i, \mathrm{~K}]=\mathrm{P}[i, \mathrm{D}]+$ $\mathrm{P}[i, \mathrm{E}]+\mathrm{P}[i, \mathrm{~F}]$; and also a probability for each frame label (?, -, o, or + ). See Fig. 1.

$\begin{array}{rrrrrrrrr}681 & \mathrm{~A} & 0.398 & * 0.601 & 0.000 & 0.000 & \mathrm{D}: \mathrm{N} * & 0.398 & * 0.601 \mathrm{~K}: \mathrm{N} * \\ 682 & \mathrm{~A} & * 0.668 & 0.000 & 0.331 & 0.000 \mathrm{~N}: \mathrm{N} . & * 0.668 & 0.331 \mathrm{~N}: \mathrm{N} \\ 683 & \mathrm{G} & * 0.548 & 0.000 & 0.000 & 0.451 & \mathrm{~N}: \mathrm{N} . & * 0.548 & 0.451 \mathrm{~N}: \mathrm{N} \\ 684 & \mathrm{~T} & 0.161 & * 0.838 & 0.000 & 0.000 & \mathrm{D}: \mathrm{N} * & 0.161 & * 0.838 \mathrm{~K}: \mathrm{N} * \\ 685 & \mathrm{~A} & * 0.593 & 0.000 & 0.405 & 0.000 & \mathrm{~N}: \mathrm{N} . & * 0.593 & 0.406 \mathrm{~N}: \mathrm{N} \\ 686 & \mathrm{~T} & 0.447 & 0.000 & 0.000 & * 0.552 & \mathrm{~F}: \mathrm{F} . & 0.447 & * 0.552 \mathrm{~K}: \mathrm{K} \\ 687 & \mathrm{~A} & 0.120 & * 0.879 & 0.000 & 0.000 & \mathrm{D}: \mathrm{D} . & 0.120 & * 0.879 \mathrm{~K}: \mathrm{K} \\ 688 & \mathrm{C} & 0.449 & 0.000 & * 0.549 & 0.000 & \mathrm{E}: \mathrm{E} . & 0.449 & * 0.550 \mathrm{~K}: \mathrm{K} \cdot \\ 689 & \mathrm{C} & 0.276 & 0.000 & 0.000 & * 0.723 \mathrm{~F}: \mathrm{F} . & 0.276 & * 0.723 \mathrm{~K}: \mathrm{K} \cdot \\ 690 & \mathrm{C} & 0.077 & 0.921 & 0.000 & 0.000 \mathrm{D}: \mathrm{D} . & 0.077 & 0.922 \mathrm{~K}: \mathrm{K} \cdot \\ \cdots & & & & & & & \end{array}$

Fig. 1. Sample output from our classifier

The first two columns of Fig. 1 are the index $i$ and the contents $b_{i}$ of each base. The next four columns show the computed probabilities $\mathrm{P}[i, \varepsilon]$ for each full label $\varepsilon \in\{\mathrm{N}, \mathrm{D}, \mathrm{E}, \mathrm{F}\}$; the most likely label is flagged with ' $*$ '. The next column has the format $X: Y Z$; where $X$ is the label $\varepsilon$ with largest $\mathrm{P}[i, \varepsilon], Y$ is the user-given "true" label $e_{i}$, and $Z$ is '.' if $X=Y$, '*' otherwise. The next three columns give similar probabilities for the weak label $c_{i}(\mathrm{~N}$ or K).

\section{Description of the Algorithm}

\subsection{Classifying Bases by Context}

In our method, the four probabilities $\mathrm{P}[i, \varepsilon]$, for each index $i$, are estimated by analyzing the base $b_{i}$ and the $p$ nearest bases on either side of it; where $p$ is a user-specifiable parameter. 
Let $W_{i}^{p}=\{i-p, i-p+1, \ldots, i+p\}$ be the window with size $k=2 p+1$ centered at position $i$; and let $\beta=b\left[W_{i}^{p}\right]$ be its (known) content. Its (unknown) true labeling $e\left[W_{i}^{p}\right]$ could be any string in $\mathcal{E}^{k}$. Our algorithm actually computes (implicitly or explicitly) a probability $\operatorname{Pr}(\eta \mid \beta)=\operatorname{Pr}\left(e\left[W_{i}^{p}\right]=\eta \mid b\left[W_{i}^{p}\right]=\beta\right.$ ) for each possible labeling $\eta \in \mathcal{E}^{k}$, taking into account its known content $\beta$. These probabilities are then combined into the four probabilities $\mathrm{P}[i, \varepsilon]$ according to the central label $\eta_{p}$ of $\eta$; that is, $\mathrm{P}[i, \varepsilon]=\sum\left\{\operatorname{Pr}(\eta \mid \beta): \eta \in \mathcal{E}^{k} \wedge \eta_{p}=\varepsilon\right\}$

\subsection{Window-Based Bayesian Inference}

In theory, the probabilities $\operatorname{Pr}(\eta \mid \beta)$ could be obtained by analysis of a training set - a reference database of labeled genomic sequences, whose labels can be assumed to be true. Specifically, we can use the approximation $\operatorname{Pr}(\eta \mid \beta) \approx$ $\#(\eta \wedge \beta) / \#(\beta)$, where $\#(\beta)$ is the the total number of windows in the training set with content $\beta$, and $\#(\eta \wedge \beta)$ is the number of windows with labeling $\eta$ and content $\beta$.

However, this approximation is accurate only if $\#(\beta)$ is sufficiently large, and is in fact useless if $\#(\beta)=0$. By a rough estimate, using this method with $k=21$ (a rather modest window size) would require a training set with more than $4^{21}=10^{12}$ correctly labeled bases - far beyond what is currently available.

To circumvent this problem, we first use Bayes's inference formula to express the "deductive" conditional probabilities $\operatorname{Pr}(\eta \mid \beta)$ in terms of the "generative" probabilities $\operatorname{Pr}(\beta \mid \eta)$. For all $\beta \in \mathcal{B}^{k}$ and all $\eta \in \mathcal{E}^{k}$

$$
\operatorname{Pr}(\eta \mid \beta)=\frac{\operatorname{Pr}(\beta \wedge \eta)}{\operatorname{Pr}(\beta)}=\frac{\operatorname{Pr}(\beta \mid \eta) \operatorname{Pr}(\eta)}{\sum_{\sigma \in \mathcal{E}^{k}} \operatorname{Pr}(\beta \mid \sigma) \operatorname{Pr}(\sigma)}
$$

\subsection{The Independence Hypotheses}

In order to apply formula (1), we need to know $\operatorname{Pr}(\beta \mid \eta)$; which is the probability that a random window of the genome with length $k=|\eta|=|\beta|$, whose true labelling is $\eta$, contains the bases $\left(\beta_{0}, \beta_{1}, \ldots, \beta_{k-1}\right)$. We also need $\operatorname{Pr}(\eta)$; which is the probability that $\eta$ is the true labeling of any $k$ consecutive bases of the genome, a priori (i.e. independently of which bases are found at those positions).

Needless to say, we cannot estimate these probabilities by counting frequencies in some training set, since the frequency approximation $\#(\beta \wedge \eta) / \#(\eta)$ is just as impractical for $\operatorname{Pr}(\beta \mid \eta)$ as $\#(\beta \wedge \eta) / \#(\beta)$ is for $\operatorname{Pr}(\eta \mid \beta)$. We get around this problem by making certain independence assumptions about the statistics of the input data. In our tests, specifically, we assumed that

H1. Within a gene, each codon is independently chosen.

H2. Within a non-coding region, each base is independently chosen.

H3. There is no correlation between the contents of distinct regions.

These assumptions seem to hold for available labeled genomes [9]. They imply, in particular, that there is no correlation between the content of coding and noncoding regions; and that any two adjacent non-coding regions can be treated as 
Table 2. Frequencies $\operatorname{Pr}(\beta \mid \mathrm{DEF})$ of codons in the exons of Reese's human genome sample. See Sec. 4.2 for details on the data set.

\begin{tabular}{|c|c|}
\hline Codon & Freq \\
\hline \hline AAA & 0.018 \\
\hline AAT & 0.013 \\
\hline AAC & 0.021 \\
\hline AAG & 0.035 \\
\hline ATA & 0.004 \\
\hline ATT & 0.012 \\
\hline ATC & 0.024 \\
\hline ATG & 0.022 \\
\hline ACA & 0.011 \\
\hline ACT & 0.011 \\
\hline ACC & 0.023 \\
\hline ACG & 0.007 \\
\hline AGA & 0.008 \\
\hline AGT & 0.008 \\
\hline AGC & 0.020 \\
\hline AGG & 0.010 \\
\hline
\end{tabular}

\begin{tabular}{|c|c|}
\hline Codon & Freq \\
\hline \hline TAA & 0.001 \\
\hline TAT & 0.010 \\
\hline TAC & 0.018 \\
\hline TAG & 0.001 \\
\hline TTA & 0.003 \\
\hline TTT & 0.013 \\
\hline TTC & 0.024 \\
\hline TTG & 0.010 \\
\hline TCA & 0.008 \\
\hline TCT & 0.011 \\
\hline TCC & 0.018 \\
\hline TCG & 0.005 \\
\hline TGA & 0.002 \\
\hline TGT & 0.008 \\
\hline TGC & 0.014 \\
\hline TGG & 0.014 \\
\hline
\end{tabular}

\begin{tabular}{|c|c|}
\hline Codon & Freq \\
\hline \hline CAA & 0.010 \\
\hline CAT & 0.008 \\
\hline CAC & 0.015 \\
\hline CAG & 0.036 \\
\hline CTA & 0.005 \\
\hline CTT & 0.010 \\
\hline CTC & 0.022 \\
\hline CTG & 0.051 \\
\hline CCA & 0.014 \\
\hline CCT & 0.018 \\
\hline CCC & 0.025 \\
\hline CCG & 0.008 \\
\hline CGA & 0.006 \\
\hline CGT & 0.005 \\
\hline CGC & 0.016 \\
\hline CGG & 0.013 \\
\hline
\end{tabular}

\begin{tabular}{|c|c|}
\hline Codon & Freq \\
\hline \hline GAA & 0.023 \\
\hline GAT & 0.018 \\
\hline GAC & 0.028 \\
\hline GAG & 0.045 \\
\hline GTA & 0.005 \\
\hline GTT & 0.008 \\
\hline GTC & 0.016 \\
\hline GTG & 0.033 \\
\hline GCA & 0.013 \\
\hline GCT & 0.019 \\
\hline GCC & 0.036 \\
\hline GCG & 0.010 \\
\hline GGA & 0.014 \\
\hline GGT & 0.012 \\
\hline GGC & 0.030 \\
\hline GGG & 0.017 \\
\hline &
\end{tabular}

a single region. On the other hand, one observes quite unequal frequencies for the 64 possible codons, as shown in Table 2 .

The irregularities of Table 2 imply significant differences in nucleotide frequencies between coding and non-coding regions. Table 3 shows the frequencies of nucleotides A, T, C, G observed in Reese's data set, in five contexts: respectively, in the first, second,

Table 3. Observed base frequencies

\begin{tabular}{|c|c|c|c|c|c|}
\cline { 2 - 6 } \multicolumn{1}{c|}{} & $\mathrm{D}$ & $\mathrm{E}$ & $\mathrm{F}$ & $\mathrm{K}$ & $\mathrm{N}$ \\
\hline $\mathrm{A}$ & 0.248 & 0.300 & 0.144 & 0.231 & 0.263 \\
\hline T & 0.160 & 0.263 & 0.184 & 0.203 & 0.278 \\
\hline C & 0.261 & 0.235 & 0.351 & 0.283 & 0.227 \\
\hline G & 0.330 & 0.200 & 0.318 & 0.283 & 0.232 \\
\hline
\end{tabular}
and third base of codons within the exons (columns D, E, F), in any position within the exons (K), and outside the exons $(\mathrm{N})$.

Note that the codon frequencies shown in Table 2 differ significantly from those that one would expect if the three letters were chosen independently with the probabilities of columns D, E, and F of Table 3. These discrepancies imply significant correlations between bases $b_{i}$ and $b_{j}$ in coding regions, whenever $|i-j| \leq 2$.

\subsection{Factoring the Productive Probabilities}

Hypotheses H1 H3 allow us to break down the factor $\operatorname{Pr}(\beta \mid \eta)$ of Formula (11) into products of probabilities of atomic events which are easier to estimate. Firstly, suppose that a window labeling $\eta$ can be split as $\eta^{\prime} \eta^{\prime \prime}$, where the last label $\varepsilon^{\prime}$ of $\eta^{\prime}$ and the first label $\varepsilon^{\prime}$ of $\eta^{\prime \prime}$ are any pair in $\mathcal{E}^{2}$ except NN, DE, EF, or FD. 
In that case, the sub-windows of the input genome corresponding to $\eta^{\prime}$ and $\eta^{\prime \prime}$ necessarily belong to distinct regions. Then, by assumption probability calculus, we have

$$
\operatorname{Pr}(\beta \mid \eta)=\operatorname{Pr}\left(\beta^{\prime} \mid \eta^{\prime}\right) \operatorname{Pr}\left(\beta^{\prime \prime} \mid \eta^{\prime \prime}\right)
$$

for any strings $\beta, \beta^{\prime}, \beta^{\prime \prime}$ such that $\left|\beta^{\prime}\right|=\left|\eta^{\prime}\right|$ and $\left|\beta^{\prime \prime}\right|=\left|\eta^{\prime \prime}\right|$. Moreover, by assumptions $\mathrm{H1}$ and $\mathrm{H2}$, Formula (3) holds also when the label pair $\varepsilon^{\prime} \varepsilon^{\prime \prime}$ is NN (two consecutive non-coding bases) or FD (the boundary between two codons).

In other words, we can split $\beta$ and $\eta$ between any two codons, any two noncoding bases, or at any place that, according to $\eta$, is obviously the boundary between two distinct regions. It follows that, for any $\beta \in \mathcal{B}^{k}$ and $\eta \in \mathcal{E}^{k}$,

$$
\operatorname{Pr}(\beta \mid \eta)=\prod_{j=1}^{m} \operatorname{Pr}\left(\beta_{j} \mid \eta_{j}\right)
$$

where

- $\eta=\eta_{1} \eta_{2} \cdots \eta_{m}$, and $\beta=\beta_{1} \beta_{2} \cdots \beta_{m}$

- for each $j, \beta_{j} \in \mathcal{B}^{\left|\eta_{j}\right|}$;

- for each $j, \eta_{j} \in\{\mathrm{N}, \mathrm{DEF}, \mathrm{DE}, \mathrm{EF}, \mathrm{D}, \mathrm{E}, \mathrm{F}\}$;

- for each $j,\left(\eta_{j}, \eta_{j+1}\right) \notin\{(\mathrm{D}, \mathrm{EF}),(\mathrm{DE}, \mathrm{F}),(\mathrm{D}, \mathrm{E}),(\mathrm{E}, \mathrm{F})\}$.

Note that the deductive probabilities $\operatorname{Pr}(\eta \mid \beta)$ cannot be factored in this way; that is the reason why we need formula (1).

\subsection{Relevant Labelings}

In principle, the Bayesian inference Formula (1) should be evaluated for all possible labelings $\eta \in \mathcal{E}^{k}$; which is obviously impractical for $k$ beyond 10 or so. Fortunately, most of those labelings turn out to be so unlikely that they can be ignored, without significant effect on the classification. More precisely, we replace Formula (1) by the approximation

$$
\operatorname{Pr}(\eta \mid \beta) \approx \frac{\operatorname{Pr}(\beta \mid \eta) \operatorname{Pr}(\eta)}{\sum_{\sigma \in Z} \operatorname{Pr}(\beta \mid \sigma) \operatorname{Pr}(\sigma)}
$$

The set of relevant labelings $Z$ is a parameter of the algorithm. As shown in Secs. 3.4 and 3.6. the algorithm's running time is proportional to $n k \# Z$, and one can get useful results with a set $Z$ of size $O(k)$.

\subsection{Estimating the a Priori Labeling Probabilities}

Besides the generative probabilities $\operatorname{Pr}(\beta \mid \eta)$, Bayes's Formula (1) also requires the a priori probabilities $\operatorname{Pr}(\eta)$ for each labeling $\eta \in \mathcal{E}^{k}$. Since the genome is assumed to consist of relatively long regions, each uniformly coding or uniformly non-coding, there is substantial correlation between the labels of adjacent bases 
- even in non-coding regions and across codon boundaries. As a consequence of these long-range correlations, there is no factoring result analogous to Formula 3 for the a priori probabilities $\operatorname{Pr}(\eta)$.

To compute $\operatorname{Pr}(\eta)$, we need to extend the genome model with information about the lengths of coding and non-coding regions, and how the former are cut out from the ancestral genes. For the tests reported below, we assumed that:

L1. Coding and non-coding regions alternate in the input string.

L2. Every region is at least $k$ bases long.

L3. The mean lengths $\mu_{\mathrm{K}}, \mu_{\mathrm{N}}$ of coding and non-coding regions are known.

L4. The first (or last) base of a coding region may have true label D, E, or F, with equal probability.

Assumptions L1 L4 greatly reduce the window labelings $\eta$ that need to be considered, from $\mathcal{E}^{k}$ to the following possibilities:

E1. The window is totally non-coding, i.e. the true labeling $\eta$ is NNN ....

E2. The window is totally coding, i.e. $\eta$ is DEFDEF . .., EFDEFD . . ., or FDEFDE ....

E3. The window straddles the boundary between two regions, one coding and one non-coding.

There is only one window labeling of type E1 three of type E2, and $6(k-1)$ of type E3 (since there are $k-1$ positions for the transition within the window, two choices for the order of $\eta^{\prime}$ and $\eta^{\prime \prime}$, and three choices for the first coding label). That makes $6 k-2$ labelings to consider for each window.

Given hypotheses L1 L4 the a priori probability $\operatorname{Pr}(\eta)$ of each labeling $\eta$ depends only on the mean lengths $\mu_{\mathrm{K}}$ and $\mu_{\mathrm{N}}$ of coding and non-coding regions. Namely, we expect one N-K transition and one K-N transition every $\mu_{\mathrm{K}}+\mu_{\mathrm{N}}$ bases, and each of these transitions will be straddled by $k-1$ windows. It follows that $\operatorname{Pr}(\eta)$ is, for each type,

$$
\text { E1 } \frac{\mu_{\mathrm{N}}-k+1}{\mu_{\mathrm{K}}+\mu_{\mathrm{N}}} \quad \text { E2 } \quad \frac{1}{3} \frac{\mu_{\mathrm{K}}-k+1}{\mu_{\mathrm{K}}+\mu_{\mathrm{N}}} \quad \text { E3 } \quad \frac{1}{6} \frac{1}{\mu_{\mathrm{K}}+\mu_{\mathrm{N}}}
$$

\section{Experimental Results}

\subsection{Test Runs}

We tested the algorithm on a set $\mathbf{H}$ of natural eukaryotic DNA sequences, as well as on a randomly generated artificial dataset $\mathbf{A}$. Both datasets were processed with windows of size $1,3,5,9,17,33,65$, and 129 .

\subsection{The Natural Dataset}

The natural dataset $\mathbf{H}$ was a collection of annotated human DNA sequences prepared by Martin Reese [11. The collection comprises 462 separate files, each 
covering one human gene (or part of one gene). After excluding a few unsuitable files (such as genes which had been tagged by software), there remained 448 files with $5,258,181$ nucleotide bases, 439,761 of them $(8.4 \%)$ belonging to exons. The average length of exon and non-exon regions were $\mu_{\mathrm{K}}=160$ and $\mu_{\mathrm{N}}=1516$, respectively.

Within exons, we set each "true" label $e_{i}$ to D, E, or F according to its position in the expressed gene (after mRNA splicing). For all other bases, we set $e_{i}$ to N. The atomic probabilities $\operatorname{Pr}(\beta \mid \eta)$ of tables 3 and 2 were obtained by counting occurrences of $\beta$ and $\eta$ in a subset of these sequences (with about 3.21 million bases), assuming the $e$ labeling as "true", as described in Sec. 3.4. The rest of the data set (about 2.05 million bases) was then classified with our algorithm, and the output was scored using the same labeling $e$ as the truth.

\subsection{The Artificial Dataset}

The artificial dataset $\mathbf{A}$ consisted of a single sequence with 45,000 nucleotide letters, obtained by concatenating coding and non-coding regions, alternately. Each region was independently generated according to hypotheses H1 H3, using the probabilities of Table 3 for each base in non-coding regions, and of Table 2 for each codon in coding regions. The first and last codons were truncated so that each coding region was equally likely to begin or end with a D, E, or F base. The length of each region was $100+\ell$ where $\ell$ was drawn from an exponential distribution, with mean 50 for coding regions and 800 for non-coding regions resulting in mean lengths $\mu_{\mathrm{K}}=150$ and $\mu_{\mathrm{N}}=900$, respectively. The true labeling $e_{i}$ of each base was recorded during its generation.

\subsection{Scoring Table and Mean Hit Score}

To evaluate the performance of the algorithm, we compared its probability estimates $\mathrm{P}[i, \varepsilon]$ for each base $b_{i}$ with the corresponding "true" label $e_{i}$. Specifically, we computed the scoring table

$$
\operatorname{SC}\left[\varepsilon, \varepsilon^{\prime}\right]=\frac{1}{n} \sum_{i=0}^{n-1}\left(e_{i}=\varepsilon^{\prime}\right) \mathrm{P}[i, \varepsilon]
$$

for all $\varepsilon, \varepsilon^{\prime} \in \mathcal{E}$; where the factor $\left(e_{i}=\varepsilon^{\prime}\right)$ is 1 if the equality holds, 0 otherwise. We can use the sum $\sigma=\sum_{\varepsilon} \operatorname{SC}[\varepsilon, \varepsilon]$ of the diagonal elements of that table as the mean hit score of the classifier.

\subsection{Test Results}

Table 4 shows the mean hit scores obtained by the algorithm on the two data sets, as a function of the window length $k$. The columns $\sigma_{\mathrm{KN}}$ and $\sigma_{\mathrm{DEF}}$ are the scores achieved on the weak $\mathrm{K} / \mathrm{N}$ classification problem and on the reading frame identification problem, respectively. 
Table 4. Hit scores for the human $(\mathbf{H})$ and artificial (A) test data

\begin{tabular}{|r||c|c||c|c|}
\hline \multicolumn{1}{|c||}{} & \multicolumn{2}{c||}{$\mathbf{H}$} & \multicolumn{2}{c|}{$\mathbf{A}$} \\
\hline$k$ & $\sigma_{\mathrm{KN}}$ & $\sigma_{\mathrm{DEF}}$ & $\sigma_{\mathrm{KN}}$ & $\sigma_{\mathrm{DEF}}$ \\
\hline 1 & 0.14 & 0.36 & 0.15 & 0.36 \\
3 & 0.17 & 0.40 & 0.18 & 0.39 \\
5 & 0.19 & 0.45 & 0.19 & 0.43 \\
9 & 0.24 & 0.55 & 0.24 & 0.49 \\
17 & 0.33 & 0.68 & 0.36 & 0.58 \\
33 & 0.52 & 0.81 & 0.54 & 0.69 \\
65 & 0.73 & 0.92 & 0.69 & 0.80 \\
129 & 0.83 & 0.98 & 0.76 & 0.86 \\
\hline
\end{tabular}

Figure 2 shows the variation of $\mathrm{P}[i, \mathrm{~K}]$, the estimated probability of $b_{i}$ belonging to a coding region, along a segment of the $\mathbf{A}$ sequence, with $k=9$ (top), $k=33$ (middle), and $k=129$ (bottom). The dashed line is the output of the ideal classifier ( 1 when $e_{i}=\mathrm{K}, 0$ when $e_{i}=\mathrm{N}$ ). Note that the coding region at bases 335-405 was was practically indistinguishable with $k=9$, but clearly and confidently detected with $k=129$.


Fig. 2. Graph of $\mathrm{P}[i, \mathrm{~K}]$ for the artificial dataset $\mathbf{A}$

\subsection{Discussion of Test Results}

While the test with natural data would seem more relevant for users, it was distorted by the use of an incorrect reference labeling. The non-exon DNA regions, which we assumed to be entirely non-coding $(\mathrm{N})$, surely contained many undocumented fossil coding regions, which we should have labeled with $\mathrm{D} / \mathrm{E} / \mathrm{F}$. This mislabeling of fossil regions had also the effect of "blurring" already small differences between the probabilities $\operatorname{Pr}(\beta \mid \eta)$ of coding and non-coding regions.

Nevertheless, the algorithm performed fairly well on the natural dataset $\mathbf{H}$, with a hit score $\sigma_{\mathrm{DEF}}=0.857$ on the frame labeling problem. While the test with artificial data $\mathbf{A}$ used the same tables $\operatorname{Pr}(\beta \mid \eta)$, which were distorted 
by the mislabeling above, the algorithm's performance was much better, with $\sigma_{\mathrm{DEF}}=0.982$ on the frame labeling problem — presumably, because the output was scored against the correct labeling. In any case, the performance obtained in both tests is only a lower bound on the performance that one could obtain with better-labeled natural training set.

\section{Conclusions and Future Work}

The main advantages of our method are consistency, correctness, and flexibility, at a modest computational cost. The use of Bayesian inference ensures that the proposed algorithm produces the best possible classification. The inclusion of coding/non-coding transitions in the Bayesian inference leads to sharp estimates for the position of those transitions for large $|w|$. The methods is easily adapted to other statistical models by changing the set of allowed window labelings $Z$, the probability tables $\operatorname{Pr}(\beta \mid \eta)$, and the a priori probabilities $\operatorname{Pr}(\eta)$.

Acknowledgements. This research was supported by CNPq (grants 301016/92-5, 304581/2004-6, 478569/2004-1) and FAPERJ/CAPES (PPPG 05/2006).

\section{References}

1. Meidanis, J., Setubal, J.C.: Introduction to Computational Molecular Biology. PWS Publishing Company (1997)

2. Batzoglou, S., Pachter, L., Mesirov, J.P., Berger, B., Lander, E.S.: Human and mouse gene structure: Comparative analysis and application to exon prediction. Genome Research (10), 950-958 (2000)

3. Fickett, J.W.: Recognition of protein coding regions in DNA sequences. Nucleic Acids Research 10(17), 5303-5318 (1982)

4. Sagot, C.M.M.F., Schiex, T., Rouzé, P.: Recognition of protein coding regions in DNA sequences. Nucleic Acids Research 30(19), 4103-4117 (2002)

5. Pertea, M., Salzberg, S.L.: Computational gene finding in plants. Plant Molecular Biology 48(1-2), 39-48 (2002)

6. Farber, R., Lapedes, A., Sirotkin, K.: Determination of eukaryotic protein coding regions using neural networks and information theory. Journal of Molecular Biology (226), 471-479 (1992)

7. Kotlar, D., Lavner, Y.: Gene prediction by spectral rotation measure: A new method for identifying protein-coding regions. Genome Research 13(8), 1930-1937 (2003)

8. Majoros, W.H., Pertea, M., Salzberg, S.L.: Efficient implementation of a generalized pair hidden Markov model for comparative gene finding. Bioinformatics 21(9), 1782-1788 (2005)

9. Staden, R., McLachlan, A.D.: Codon preference and its use in identifying protein coding regions in long DNA sequences. Nucleic Acids Res. 10(1), 141-155 (1982)

10. Capua, R.O., da Gama Leitão, H.C., Stolfi, J.: Uma abordagem estatística para identificação de éxons. In: WOB 2004, Brasília, DF (2004)

11. Reese, M.: Database with human genome sequences (2005), http://www.fruitfly. org/seq_tools/datasets/Human/multi_exon_GB.dat 